\title{
Prevalence of Musculoskeletal Pain and Assessment of Ergonomic Factors in Undergraduate Dental Students: An Observational Study
}

\author{
${ }^{1} \mathrm{R}$ Vinaya Kumar, ${ }^{2} \mathrm{P}$ Anuroopa, ${ }^{3} \mathrm{MS}$ Nalini, ${ }^{4} \mathrm{~S}$ Savita
}

\begin{abstract}
Background: The musculoskeletal health of dental professionals has been the focus of several studies globally. It has been estimated that work-related musculoskeletal injuries occur in 54 to $93 \%$ of dental professionals. However, when it comes to studies carried out on dental students, there is a dearth of information. Therefore, the objectives of this study were to determine the ergonomic factors and presence of muscular pain in dental students undergoing clinical training at Rajarajeswari Dental College and Hospital, Bengaluru, India.
\end{abstract}

Materials and methods: Data collection was carried out on a sample of 103 students pursuing their undergraduate program in dentistry using a structured questionnaire comprising questions pertaining to the recipient's sociodemographic information, ergonomic features, medical information, musculoskeletal disorders (MSDs) resulting from clinical practice, musculoskeletal demands, posture related queries, workplace, instruments and exercises.

Results: The results of this study revealed a high prevalence of musculoskeletal pain in dental students. Further, the study also highlighted the lack of knowledge among the students which could have led to the aggravation of symptoms.

Conclusion: Therefore, it is essential to include ergonomics in the dental curriculum so as to educate students regarding adoption of healthy postures and subsequently reduce the risk of MSDs. The etiology of musculoskeletal disease is multifactorial and consequently preventive strategies should focus on ergonomics, breaks at work, general health and physical exercise.

Keywords: Dentists, Ergonomics, Musculoskeletal disorders.

How to cite this article: Kumar RV, Anuroopa P, Nalini MS, Savita S. Prevalence of Musculoskeletal Pain and Assessment of Ergonomic Factors in Undergraduate Dental Students: An Observational Study. J Health Sci Res 2014;5(2):1-5.

Source of support: Nil

Conflict of interest: None

\footnotetext{
${ }^{1-3}$ Reader, ${ }^{4}$ Professor and Head

${ }^{1-4}$ Department of Periodontology, Rajarajeswari Dental College and Hospital, Bengaluru, Karnataka, India
}

Corresponding Author: R Vinaya Kumar, Reader Department of Periodontology, Flat 402, Sri Kiran Residency No.14, 4th Cross, 30th Main, Banashankari 3rd Stage Bengaluru-560085, Karnataka, India, Phone: 9844301016 e-mail: drrvinayakumar@gmail.com

\section{INTRODUCTION}

A definitive link between fixed postures and musculoskeletal disorders (MSDs) (including pain, weakness and paresthesia) has been documented for a number of occupations. ${ }^{1}$ Dentistry is a profession that generally produces muscular pain and soreness, which are usually innocuous and slow to appear. As a result, the symptoms are usually ignored until they become chronic and permanent lesions. ${ }^{2}$

The term work-related MSDs refers to disorders to which the work environment contributes significantly or to disorders that are made worse or longer lasting by work conditions or workplace risk factors. ${ }^{3}$ They are characterized by the presence of discomfort, disability or persistent pain in the joints, muscles, tendons and other soft parts, caused or aggravated by repeated movements and prolonged awkward or forced body postures. ${ }^{4}$

The specific character of dental work is connected with and accompanied by onerous and harmful effects. Uncomfortable positions assumed by dentists during work undoubtedly cannot remain without affecting health. Standing or sitting positions which are frequently adopted while working can lead to twisting of the spine and straining of tissues which could be the source of painful disorders and diseases of the musculoskeletal system and the peripheral nervous system. ${ }^{5}$

The musculoskeletal health of dental professionals has been the subject of numerous studies worldwide, and their focus has been on the pain experienced by the practitioner. ${ }^{6}$ Musculoskeletal pain, particularly back pain, has been found to be a major health problem for dental practitioners. ${ }^{7}$ Biller found that $65 \%$ of dentists complained of back pain. Even after the evolution to seated four-handed dentistry and ergonomic equipment, studies found back, neck, shoulder or arm pain present in up to $81 \%$ of dental operators. ${ }^{6}$ In fact, it has been estimated that work-related musculoskeletal injuries occur in 54 to $93 \%$ of dental professionals, with the most frequent injuries occuring in the spine (neck and back), shoulders, elbows and hands. ${ }^{8}$

Despite this grim scenario, occupational health programs are not being carried out in a satisfactory manner nor is the adequate training of these activities being 
promoted. ${ }^{2}$ While many studies have been carried out where the study population comprised of dental professionals, there is a paucity of information when it comes to studies carried out on dental students. Hence, we felt that the target population should actually be the students as they acquire detrimental habits and practices during the course of their training which can have a deleterious effect on their health in the long run. Therefore, it becomes necessary to find out if there is a presence or absence of muscular pain and the possible associated ergonomic factors in dental students, in order to implement preventive and corrective measures resulting in promotion of healthy lifestyles.

Thus, the objectives of this study were to determine the ergonomic factors and presence of muscular pain in dental students undergoing clinical training at Rajarajeswari Dental College and Hospital, Bengaluru, India.

\section{MATERIALS AND METHODS}

The questionnaire study was carried out on a sample of 103 students pursuing their undergraduate program in dentistry at Rajarajeswari Dental College and Hospital, Bengaluru, India who met the following inclusion criteria: students pursuing III and IV year Bachelor of Dental Surgery (BDS) course at the institution and who volunteered to participate in the study. The exclusion criteria were as follows: students suffering from previously diagnosed scoliosis, chronic lumbar pain, those who also performed other work activities requiring forced postures, who had been subjected to disk hernia surgery, history of fractures in the trunk or lower members, history of muscular tear of lower members, students who practiced sports at least three times a week.

Data collection was carried out using a structured questionnaire. The questionnaire comprised of questions pertaining to the recipient's sociodemographic information (age, gender, etc.), ergonomic features (height, weight, dominant limb, working hours, working chair characteristics, etc.), medical information, MSDs resulting from clinical practice, musculoskeletal demands, posture related queries, workplace, instruments and exercises. Prior to being finalized, the questionnaire was pilottested on a group of six practicing students to ensure clarity and validity. The questionnaire was designed so that it would take approximately around 10 minutes to complete. Following completion of the questionnaire, the students were interviewed to clarify any confusion and provide missing data, if any.

\section{RESULTS}

The questionnaire was completed by 103 third and fourth year BDS students. The sample population comprised 21 males and 82 females and the average age was 21.4 years (19-29 years).

Characteristics of working chair: In order to assess the students' awareness, few questions were included in the questionnaire regarding the working chair. When questioned about the backrest, 81 out of the 103 students replied that their working chair had a backrest while 17 answered that their working chair did not have one and the remaining $5 \mathrm{did}$ not answer the question. This was despite the fact that all the working chairs in the institution had a backrest. Only 40 students used the backrest while working. Fifty eight students felt that the dental stool they were using was ergonomically regulated for individual needs. Sixty-five percent of students positioned their chair to maintain an ergonomic posture while practicing.

Presence of pain: Ninety-one percent of the students reported muscular pain due to clinical practice (Graph 1). Students experienced more pain while performing periodontics and restorative procedures, while least pain was observed with respect to the specialty of prosthodontics (Graph 2). This could probably be attributed to the fact that the former specialties mainly involve working in a sitting posture while for the latter, work can be accomplished mainly by standing and to a lesser extent using a sitting posture. Further, most pain reported in the specialty of periodontics could be due to the fact that the clinical work at undergraduate level involves hand scaling.

Areas affected with pain: The neck was the most frequently affected area (52.4\% of students), followed by pain in the lumbar area (42.7\%), dorsal area (39.8\%), shoulders (31.1\%), hand (28.2\%), wrist (25.2\%), arm (20.4\%), cervical zone $(18.4 \%)$ and the least affected being the forearm $(13.6 \%)$ (Graph 3). In turn, $24.5 \%$ of the students suffered pain in a single location, $22.3 \%$ in two locations and $53.2 \%$ in three or more locations.

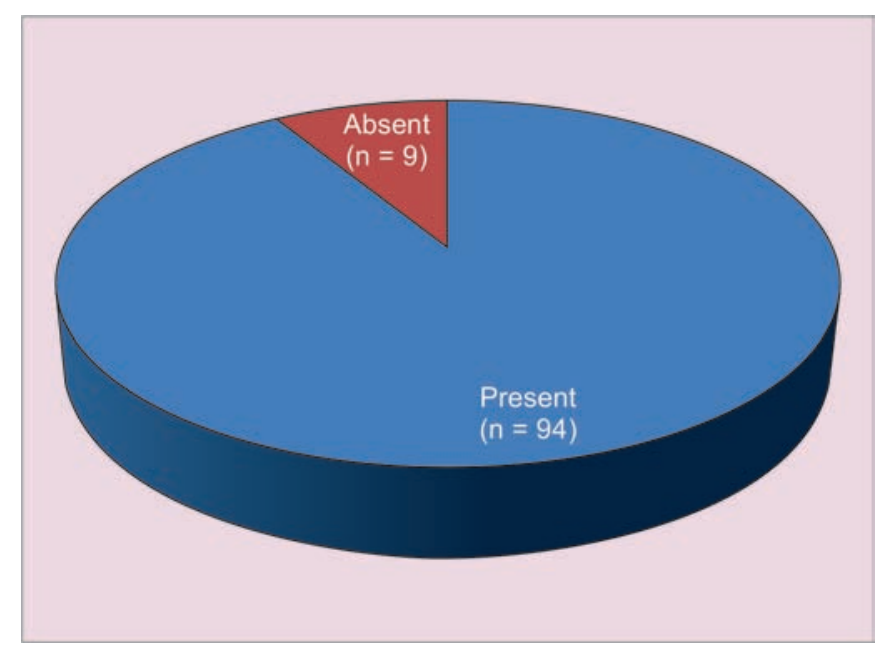

Graph 1: Prevalence of muscular pain 


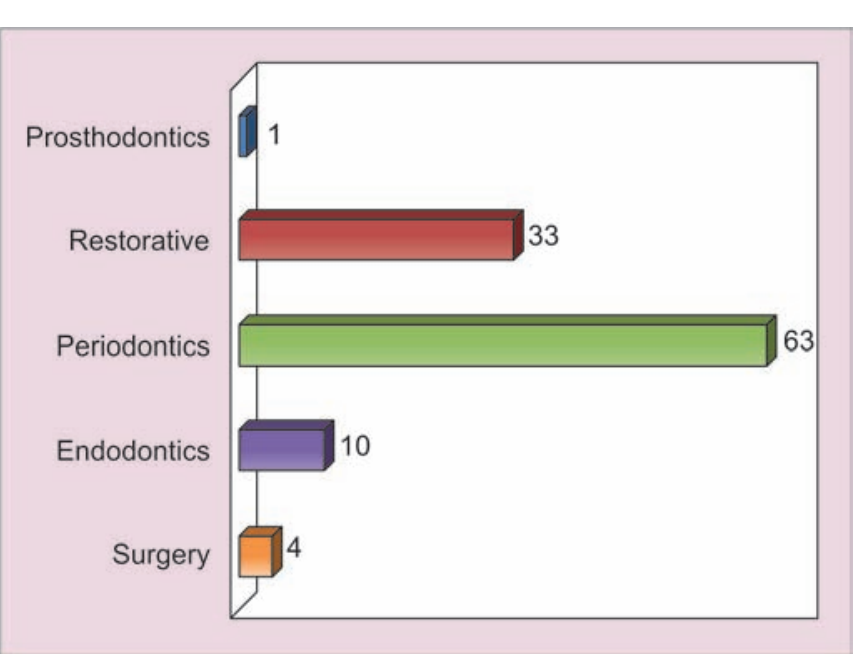

Graph 2: Clinical activities producing muscular pain distributed based on specialties

Physician consultation: Despite a high percentage of students suffering from pain, only $10.6 \%$ of them had consulted a physician for the same (Graph 4).

Musculoskeletal demands: A majority of the respondents agreed that their work involved frequent, repetitive and forceful motions; sustained muscle contraction and frequent bending of the neck, shoulder, elbow, wrist or finger joints (Table 1).

Posture related queries: $75.7 \%$ of the students claimed to change their work posture during clinical practice, while $70.9 \%$ of them reported that the instruments were easily available within hand reach without making strenuous movements. It was also found that the majority of students worked with their legs slightly separated and supported themselves on the sole of their feet (Table 1).

However, a large proportion of students responded that they did not perform torsions or cervical flexions in order to improve vision when working in the oral cavity. $50.5 \%$ of students reported that they maintained a seating

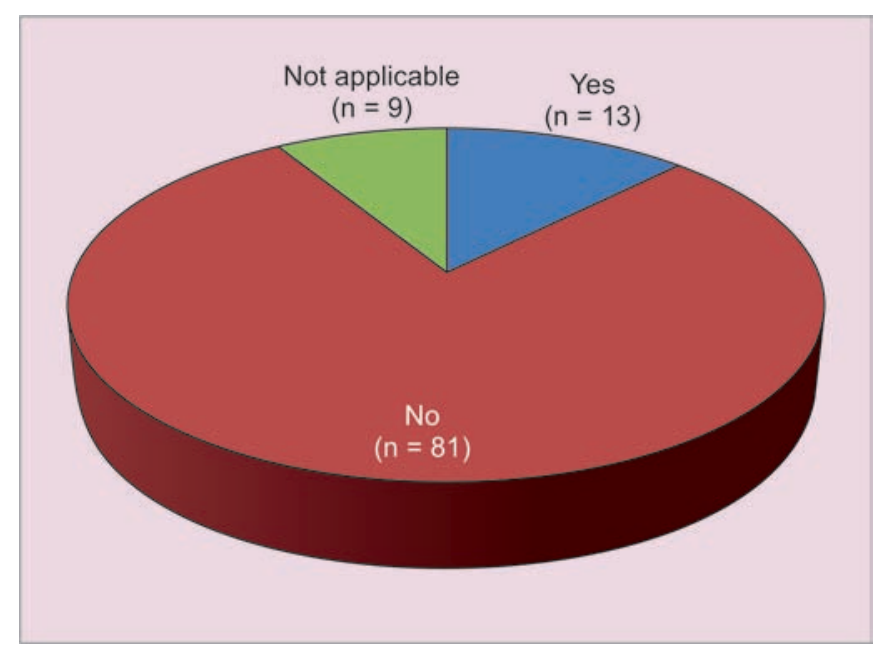

Graph 4: Student distribution of physician consultation

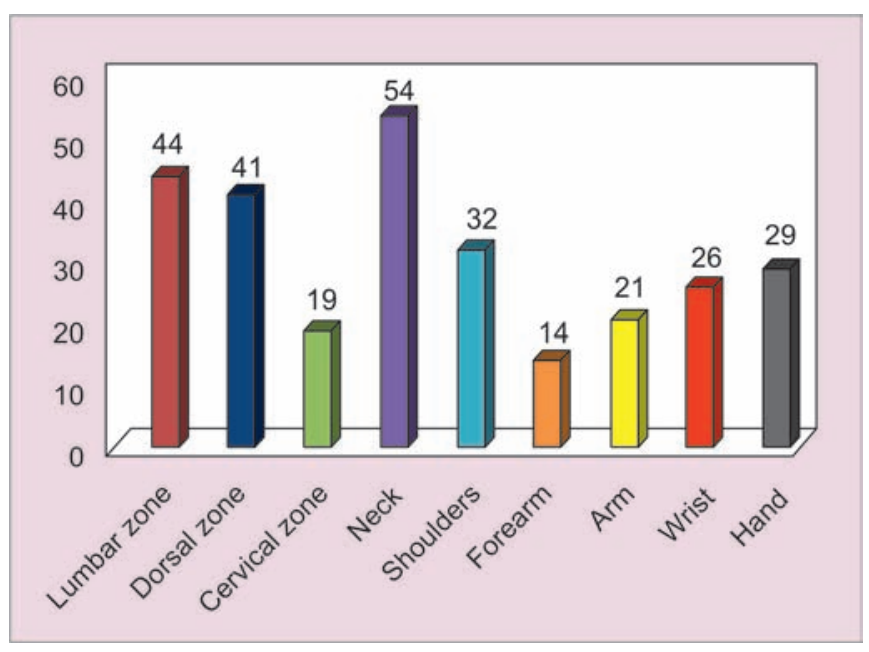

Graph 3: Areas/zones affected by pain

position in the middle of the stool, while $69.9 \%$ did not keep their arms at sternum height when working resulting in lumbar inclination of the spine (Table 1).

Condition of the workplace: A majority of students considered that they have sufficient space and light at their workplace. However, $25.2 \%$ of students felt that the excessive noise at the workplace was annoying or distracting (Table 1).

Exercises: In our study, we found that although the students experienced muscular pain, a large number did not perform any stretching exercises after finishing practice which could have helped in alleviating the symptoms (Graph 5).

\section{DISCUSSION}

Almost all published studies on musculoskeletal problems in dentistry have an observational design. Studies using questionnaires, as in our case, are useful for identifying the prevalence of a disorder, determining the clinical features of patients, and for designing possible

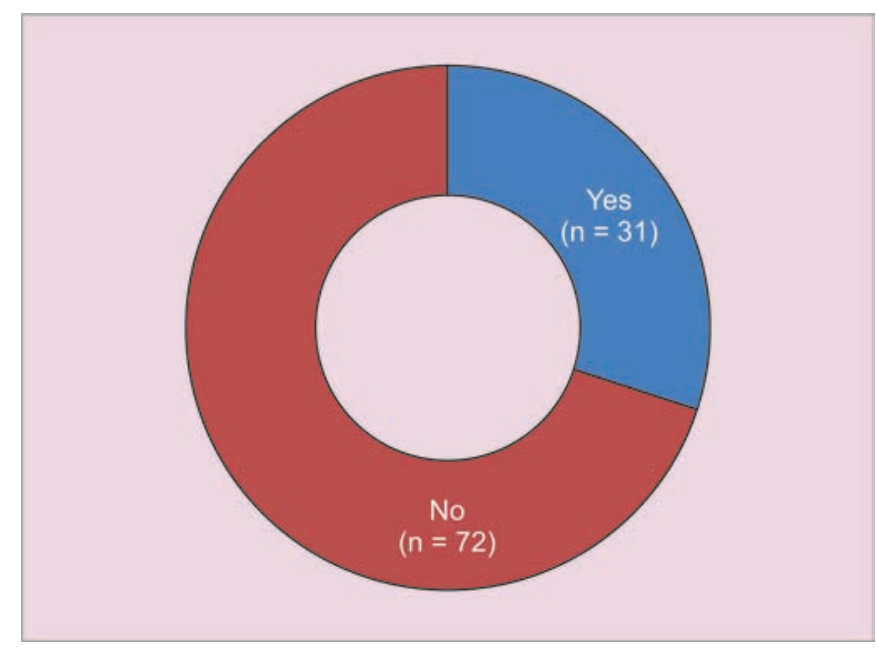

Graph 5: Details of students performing stretching exercises after practice 
Table 1: Variables assessed in the study

\begin{tabular}{llll}
\hline Questions & Yes [n (\%)] & No [n (\%)] & Not answered [n (\%)] \\
\hline Musculoskeletal demands & & & \\
Perform frequent, repetitive, forceful motions & $70(68 \%)$ & $28(27.2 \%)$ & $5(4.8 \%)$ \\
Postures involve sustained muscle contraction of limb & $64(62.1 \%)$ & $30(29.1 \%)$ & $9(8.8 \%)$ \\
Frequent bending of neck shoulder, elbow, wrist or finger joints & $86(83.5 \%)$ & $12(11.6 \%)$ & $5(4.9 \%)$ \\
Able to change work posture, seating or standing during practice & $78(75.7 \%)$ & $24(23.3 \%)$ & $1(1 \%)$ \\
Instruments within hand reach without making strenuous movements & $73(70.9 \%)$ & $22(21.3 \%)$ & $8(7.8 \%)$ \\
Posture related queries & & & $1(1 \%)$ \\
Cross your legs while working & $11(10.7 \%)$ & $91(88.3 \%)$ & $0(0 \%)$ \\
Work with legs slightly separated & $91(88.3 \%)$ & $12(11.7 \%)$ & $1(1 \%)$ \\
Support yourself on sole of your feet while working & $74(71.8 \%)$ & $28(27.2 \%)$ & $4(3.9 \%)$ \\
Put your elbows and arms on the side of your ribs & $27(26.2 \%)$ & $72(69.9 \%)$ & $2(1.9 \%)$ \\
Keep arms at sternum height when working & $29(28.2 \%)$ & $72(69.9 \%)$ & $0(0 \%)$ \\
Workplace & & & $0(0 \%)$ \\
Enough space & $89(86.4 \%)$ & $14(13.6 \%)$ & $0(0 \%)$ \\
Sufficient light & $94(91.3 \%)$ & $9(8.7 \%)$ & $77(74.8 \%)$ \\
Excessive noise & $26(25.2 \%)$ & & $5(4.8 \%)$ \\
Instruments & & & $6(5.8 \%)$ \\
Instruments in optimal condition & $73(70.9 \%)$ & $25(24.3 \%)$ & $1(1 \%)$ \\
Handle too small or too large & $16(15.5 \%)$ & $81(78.7 \%)$ & $61(59.2 \%)$ \\
Handle shape causing operator to bend wrist during use & $41(39.8 \%)$ & & $3(0 \%)$ \\
Exercises & & $72(69.9 \%)$ & $3.9 \%)$ \\
Perform stretching exercises & $31(30.1 \%)$ & $70(68 \%)$ & $(29.1 \%)$ \\
\hline
\end{tabular}

preventive strategies despite their limitations like difficulty in identifying risk factors and questionable utility for diseases of low incidence and short duration. ${ }^{4}$

Sim, Lacey and Lewis (2006) established that there is a positive relationship between rigid postures and muscular skeletal disorders in different professions which could include pain, weakness, and paresthesia. This has been widely documented and studied for a large number of professions. ${ }^{2}$ Dentists are normally included within the group of professionals at risk of suffering musculoskeletal disorders, due to prolonged awkward or forced postures at work and failure to adopt preventive measures. ${ }^{4}$ Forced postures are frequently adopted by the dental students leading to physiological alterations or muscular skeletal disorders which generate back and neck pain, among others. ${ }^{2}$

Our study found that most students exhibited some kind of musculoskeletal pain, in coincidence with the results of the study conducted by Diaz-Caballero et al, ${ }^{2}$ and Harutunian et al. ${ }^{4}$ In agreement with different studies, the region most commonly affected by pain was the neck, followed by the lumbar zone as observed by Leggat and Smith. ${ }^{7}$

As in our study, most authors found that females were more susceptible to this type of pain, although the reason is ambiguous. Some authors attributed this difference to a lesser muscle tone and a higher incidence of osteoporosis among women. The role of age is even more debatable. While there are studies that assert that the frequency of pain remains stable with age, ${ }^{9}$ others believe that musculoskeletal discomfort is highest around the sixth decade of life. A third group considers that discomfort is greater in young professionals. In our study, we found young professionals to have a higher incidence of neck pain. This could be due to incorrect working positions, since older dentists employ more indirect vision and usually avert neck overload. ${ }^{4}$

In this study, we found that the students, although enduring musculoskeletal discomfort, did not take measures to prevent or lessen the symptoms. Surprisingly, only 10 out of 94 students suffering from pain consulted a physician for the problem. More alarming was the fact that three of the respondents resorted to self medication and treatment, which could have had deleterious effects.

\section{CONCLUSION}

Muscular pain is a widespread problem in dentists which commences at the time they start their professional studies and it stays with them during their professional practice affecting the spine, neck, shoulders, hands and various other areas.

The results of this study reveal that the prevalence of musculoskeletal pain in dental students is high. Dental studies and dental work often entail time spent in static, 
uncomfortable positions, which can cause musculoskeletal symptoms even over the relatively short clinical training phase.

Further, the study also highlighted the lack of knowledge about ergonomics among the students which could have led to the aggravation of symptoms. Therefore, it becomes absolutely imperative to include and teach ergonomics in the curriculum, thereby facilitating the adoption of healthy postures by the students and consequently reducing the risk of MSDs.

Preventing chronic pain in dentistry may require a paradigm shift within the profession regarding clinical work habits, including proper use of ergonomic equipment, frequent short stretch breaks and regular strengthening exercise.

To conclude, further studies need to be undertaken to recognize the causes of MSD and to identify appropriate interventions to help reduce the prevalence of MSDs.

\section{REFERENCES}

1. Marshall ED, Duncombe LM, Robinson RQ, Kilbreath SL. Musculoskeletal symptoms in New South Wales Dentists. Aust Dent J 1997;42(4):240-246.
2. Diaz-Caballero AJ, Gómez-Palencia IP, Díaz-Cárdenas S. Ergonomic factors that cause the presence of pain muscle in students of dentistry. Med Oral Patol Oral Cir Bucal 2010; 15(6):e906-911.

3. Gupta S. Ergonomic applications to dental practice. Ind J Dent Res 2011;22(6):816-822.

4. Harutunian K, Gargallo-Albiol J, Figueiredo R, GayEscoda C. Ergonomics and musculoskeletal pain among postgraduate students and faculty members of the School of Dentistry of the University of Barcelona (Spain). A crosssectional study. Med Oral Patol Oral Cir Bucal 2011;16(3): e425-429.

5. Szymanska J. Disorders of the musculoskeletal system among dentists from the aspect of ergonomics and prophylaxis. Ann Agric Environ Med 2002;9(2):169-173.

6. Valachi B, Valachi K. Mechanisms leading to musculoskeletal disorders in dentistry. J Am Dent Assoc 2003;134(10): 1344-1350.

7. Leggat PA, Smith DR. Musculoskeletal disorders selfreported by dentists in Queensland, Australia. Aust Dent J 2006;51(4):324-327.

8. Sudarshan R, Ganesan SV. Ergonomics in dentistry-a review. J Environ Occup Sci 2012;1(2):125-128.

9. Lehto TU, Helenius H, Alaranta HT. Musculoskeletal symptoms of dentists assessed by a multidisciplinary approach. Community Dent Oral Epidemiol 1991;19(1):38-44. 